Research Article

\title{
Fatigue Life Analysis of Aluminum Alloy Notched Specimens under Non-Gaussian Excitation based on Fatigue Damage Spectrum
}

\author{
Kuanyu Chen $\mathbb{D}^{1,3}$ Guangwu Yang $\mathbb{D}^{1,},{ }^{1}$ Jianjun Zhang, ${ }^{2}$ Shoune Xiao $\mathbb{D}^{1},{ }^{1}$ and Yang Xu ${ }^{1}$ \\ ${ }^{1}$ State Key Laboratory of Traction Power, Southwest Jiaotong University, Chengdu 610031, China \\ ${ }^{2}$ Materiel Service Product Department Avic China Aero-Polytechnology Establishment, Beijing 101400, China \\ ${ }^{3}$ Comac Shanghai Aircraft Design and Research Institute, Shanghai 201210, China
}

Correspondence should be addressed to Guangwu Yang; 191797203@qq.com

Received 25 June 2021; Revised 10 September 2021; Accepted 8 October 2021; Published 28 October 2021

Academic Editor: Xavier Chiementin

Copyright ( 2021 Kuanyu Chen et al. This is an open access article distributed under the Creative Commons Attribution License, which permits unrestricted use, distribution, and reproduction in any medium, provided the original work is properly cited.

In this study, a non-Gaussian excitation acceleration method is proposed, using aluminum alloy notched specimens as a research object and measured acceleration signal of a certain airborne bracket, during aircraft flight as input excitations, based on the fatigue damage spectrum (FDS) theory. The kurtosis and skewness of the input signal are calculated and the non-Gaussian characteristics and amplitude distribution are evaluated. Five task segments obey a non-Gaussian distribution, while one task segment obeys a Gaussian distribution. The fatigue damage spectrum calculation method of non-Gaussian excitation is derived. The appropriate FDS calculation method is selected for each task segment and the acceleration parameters are set to construct the acceleration power spectral density, which is equivalent to the pseudo-acceleration damage. A finite-element model is established, the notch stress concentration factor of the specimen is calculated, the large mass point method is used to simulate the shaking table excitation, and a random vibration analysis is carried out to calculate the accelerated fatigue life. The simulation results show that the relative error between the original cumulative damage and test original fatigue life is $15.7 \%$. The shaking table test results show that the relative error of fatigue life before and after acceleration is less than $16.95 \%$, and the relative error of test and simulation is $24.27 \%$. The failure time of the specimen is accelerated from approximately $12 \mathrm{~h}$ to $1 \mathrm{~h}$, the acceleration ratio reaches 12 , and the average acceleration ideal factor is 1.125 , which verifies the effectiveness of the acceleration method. It provides a reference for the compilation of the load spectrum and vibration endurance acceleration test of other airborne aircraft equipment.

\section{Introduction}

The bench tests of airborne equipment are divided mainly into functional vibration tests and vibration endurance acceleration tests. The vibration endurance acceleration test can reproduce the fatigue damage during the whole service life in a relatively short time, which is of significance. Compared to the outfield environment test, the continuous acceleration bench test is repeatable, which can largely reduce the labor and time costs.

Therefore, to carry out the acceleration fatigue life bench test of airborne equipment, compilation of the frequency domain synthetic acceleration spectrum has become a key step in the vibration endurance bench test. The selection of acceleration parameters and determination of the equivalent acceleration time directly affect the rationality of the acceleration fatigue life. In engineering problems, many loads exhibit obvious non-Gaussian characteristics, particularly under harsh working conditions, while the non-Gaussian characteristics of excitation are particularly obvious [1]. However, in the analysis, we usually assume that the random load on the structure obeys a stationary Gaussian distribution, which often leads to the danger of fatigue damage, which implies a hidden danger to the equipment service [2]. The most commonly used acceleration method is the frequency domain synthesis method based on the fatigue damage spectrum (FDS), proposed in French military standards to evaluate the potential damage of different components under 
dynamic excitation [3]. Since then, extensive related studies have been carried out. Liu [4] applied super-Gaussian basic excitation to a cantilever beam, studied its dynamic response and structural fatigue life, and deduced Gaussian and superGaussian vibration acceleration models. Cheng [5] established an analytical expression for a non-Gaussian probability density function, combined a probabilistic power spectrum with the Dirlik formula, proposed a formula for the calculation of non-Gaussian wide- and narrow-band fatigue lives, and provided a random vibration acceleration test scheme based on the failure mechanism. According to the actual operation of subway vehicles, Wang [6] carried out road spectrum simulation tests, simulated long-life acceleration spectrum tests, and standard spectrum tests based on the FDS synthetic acceleration power spectral density (PSD). The critical surface method based on the maximum principal stress and shear stress criterion was used to predict the multiaxial acceleration fatigue life. Based on the equivalent principle of fatigue damage, Qin et al. [7] converted the nonGaussian excitation into the frequency-domain PSD for the shaking table test, so that the vibration environment of the traction converter is simulated more accurately. In 2014, Lalanne [8] studied the different calculation methods and influence parameters of the extreme response spectrum (ERS) and FDS based on a single-degree-of-freedom (SDOF) system. Wolfsteiner [9] employed the high-order spectrum to calculate the FDS of a multi-degree-of-freedom (MDOF) system to estimate the damage of the system under nonGaussian excitation. Wen [10] employed the load signal of the front axle of an $88-\mathrm{kW}$ tractor, considering the effects of load amplitude and material fatigue, and proposed an accelerated durability testing method based on PSD FDS editing (PDLSD). This ensured that the accelerated endurance test could reproduce the fatigue load characteristics of the tractor assembly. Based on FDS and test synthesis method, Cianetti [11] compared the fatigue damages caused by acceleration excitation under different conditions and verified the proposed acceleration method by a durability test case.

Conventional vibration endurance acceleration tests usually ignore the transfer process from excitation to response and non-Gaussian characteristics of excitation signals [12]. At present, there is no good acceleration method for the acceleration of non-Gaussian and Gaussian mixed excitation, and the selection of acceleration parameters for non-Gaussian excitation is only based on experience. It is impossible to get an accurate accelerated excitation PSD applied to the shaking table test. In this study, considering the measured non-Gaussian excitation of an airborne equipment, mounting bracket, during aircraft flight as an input, a non-Gaussian excitation acceleration method based on the FDS is proposed. The calculation method of fatigue damage spectrum is summarized, the advantages and disadvantages of each method are compared, the influence of acceleration parameters on accelerated PSD is explored, and the appropriate acceleration parameters are selected to deal with non-Gaussian excitation. Finally, simulations and experiments are carried out to verify the effectiveness of the accelerated method and the accuracy of the finite element model.

\section{Theoretical analysis and accelerated fatigue model}

In order to edit the input load spectrum in frequency domain or time domain, it is necessary to distinguish the nonGaussian or Gaussian characteristics of the input excitation. Then the fatigue damage spectrum of each working condition or task section is calculated based on different FDS calculation methods and synthesized by linear superposition. Finally the accelerated PSD used on the bench vibration test is calculated by acceleration formula based on the principle of damage equivalence.

\subsection{Discriminant principle of non-Gaussian characteristics.} The PSD is a second-order statistic. For a stationary Gaussian vibration with a mean value of 0 , the PSD can include the statistical characteristics of random vibrations [13]. For non-Gaussian random processes, the information contained in the PSD is insufficient. The load suffered by the equipment in aircraft operation is generally non-Gaussian excitation; therefore, it is necessary to distinguish nonGaussian parts from a input excitation. For a random process $X(t)$, skewness $S$ and kurtosis $K$ are commonly used in engineering to calculate third-order and forth-order random vibration statistics. The calculation equation is shown as (1) and (2):

$$
\begin{aligned}
& K=\frac{E[X-E(X)]^{4}}{\left\{E[X-E(X)]^{2}\right\}^{2}}, \\
& S=\frac{E[X-E(X)]^{3}}{\left\{E[X-E(X)]^{2}\right\}^{3 / 2}} .
\end{aligned}
$$

In engineering, when the kurtosis of a random signal is $K$ $=3$ and $S=0$, the random excitation is a Gaussian process, when $K>3$, the random excitation is a super-Gaussian excitation, and when $K<3$, it is a sub-Gaussian random excitation [14]. Additionally, to obtain the amplitude distribution properties of input excitations, the cycles times of excitation amplitude intervals are counted and contrasted with the Gaussian distribution to see whether the excitation amplitudes obey a Gaussian distribution.

\subsection{Fatigue damage spectrum calculation methods}

\subsubsection{Gaussian frequency domain fatigue damage models.} The estimation methods of fatigue life of random vibration are generally divided into time domain method and frequency domain method. In the frequency domain method, the response stress power spectral density (PSD) is used to predict the fatigue life of engineering structures by its spectral parameters, the $S-N$ curve of materials and the appropriate damage theory. In this process, the spectral parameters describing the statistical characteristics of the random process, which avoids directly dealing with the complex stress time history and greatly shortens the time and workload are only be calculated in the frequency domain method. 
For Gaussian stochastic processes, the power spectral density function $G(f)$ is used to describe the frequency domain characteristics of excitation. Meanwhile, the statistical characteristics of the power spectral density function can be expressed by the moment [15]. Given a random signal $X(t)$, the moment of order $n$ (close to the origin) is the quantity:

$$
\begin{aligned}
M_{n} & =E\left\{\left[\frac{\mathrm{d}^{n / 2} X(t)}{\mathrm{d} t^{n / 2}}\right]^{2}\right\} \\
& =\lim _{T \rightarrow \infty} \frac{1}{2 T} \int_{-T}^{+T}\left[\frac{\mathrm{d}^{n / 2} X(t)}{\mathrm{d} t^{n / 2}}\right]^{2} \mathrm{~d} t .
\end{aligned}
$$

Expressed in the form of power spectral density $G(f)$, which can be written as:

$$
m_{n}=\int_{0}^{\infty} f^{n} G(f) \mathrm{d} f, \quad n=1,2,3 \ldots
$$

The moment of order zero is none other than the square of the rms value $X_{\mathrm{rms}}(t)$ :

$$
\begin{aligned}
M_{0} & =E\left[X^{2}(t)\right] \\
& =\lim _{T \longrightarrow \infty} \frac{1}{2 T} \int_{-T}^{+T} X^{2}(t) \mathrm{d} t=X_{\mathrm{rms}}^{2}, \\
M_{0} & =R(0) \\
& =\int_{0}^{\infty} G(f) \mathrm{d} f .
\end{aligned}
$$

Moments of order $\mathrm{n}$ are related to stochastic processes and their derivatives as follows:

$$
\left\{\begin{array}{c}
m_{0}=\sigma_{x}^{2}=E\left[x^{2}(t)\right], \\
m_{2}=\sigma_{\dot{x}}^{2}=E\left[\dot{x}^{2}(t)\right], \quad=E\left[\ddot{x}^{2}(t)\right] . \\
m_{4}=\sigma_{\ddot{x}}
\end{array}\right.
$$

The zero crossing frequency $E(0)$ (the number of times the zero is crossed from the bottom up) and the peak frequency $E(p)$ (the number of peaks in the sample) can be approximately estimated by the moment as follows:

$$
\begin{aligned}
& E[0]=\sqrt{\frac{m_{2}}{m_{0}}}, \\
& E[p]=\sqrt{\frac{m_{4}}{m_{2}}} .
\end{aligned}
$$

Random vibration theory introduces irregular factor $\gamma$ and spectral width coefficient $\varepsilon$ to distinguish whether a stationary random process is a narrowband process or a broadband process.

$$
\begin{aligned}
\gamma & =\frac{m_{2}}{\sqrt{m_{0} m_{4}}} \\
& =\frac{E[0]}{E[p]} \in(0,1), \\
\varepsilon & =\sqrt{1-\gamma^{2}} \in(0,1) .
\end{aligned}
$$

When $\varepsilon \longrightarrow 0$ or $\gamma \longrightarrow 1$, the stochastic process is a narrow-band process; when $\varepsilon \longrightarrow 1$ or $\gamma \longrightarrow 0$, the stochastic process is a broadband process, especially at that time $\gamma=0$, the stochastic process can be regarded as white noise. In engineering, it is generally believed that the amplitude probability density function of narrowband random process tends to Rayleigh distribution when $0 \leq \varepsilon \leq 0.3$ or when $0.7 \leq \gamma \leq 1$. When $0.7 \leq \varepsilon \leq 1$ or $0 \leq \gamma \leq 0.3$, the excitation can be regarded as a broadband stochastic process, and there are many models that can be described in fatigue theory [16]. The amplitude time history of narrow band and broad band random vibration is shown in the Figure 1 .

For continuous time histories of random stress, the general expression of fatigue damage can be written in the form of the following integral [17]:

$$
\begin{aligned}
D & =\int_{0}^{+\infty} \frac{N \rho(\sigma)}{C \sigma^{-m}} \mathrm{~d} \sigma \\
& =\frac{N_{0} T}{C} \int_{0} \sigma^{m} \rho(\sigma) \mathrm{d} \sigma,
\end{aligned}
$$

where $\rho(\sigma)$ denotes the probability density function of random stress response on the specimen, $\mathrm{T}$ is the total time of exposure to the random vibration excitation, $N_{0}$ is the average number of the zero up-crossings per unit time in the stress time history. If the stress response obeys a narrowband distribution, i.e., the irregular coefficient $r$ tends to 1 [18], the expression of probability density function is as follows:

$$
\rho(\sigma)=\frac{\sigma}{\sigma_{\mathrm{rms}}} \exp \left(\frac{-\sigma^{2}}{2 \sigma_{\mathrm{rms}}^{2}}\right),
$$

where $\sigma_{\text {rms }}$ is the root mean square of stress response. Then Substitute (10) into (9) to obtain

$$
D=\frac{K^{b}}{C} n_{0}^{+} T\left(\sqrt{2} z_{\mathrm{rms}}\right)^{b} \Gamma\left(1+\frac{b}{2}\right) .
$$

The above equation uses the Rayleigh distribution as an amplitude probability density function. The rms displacement response $z_{\text {rms }}$ to a vibration can be further simplified. If the PSD is defined with respect to frequency f, the $z_{\text {rms }}$ can be written as:

$$
z_{\mathrm{rms}}=\sqrt{\frac{\omega_{0}}{4} Q G_{X_{0}}(f)}
$$

and when the input is an acceleration, the simplified $z_{\mathrm{rms}}$ is

$$
z_{\mathrm{rms}}=\sqrt{\frac{Q G_{\ddot{x}_{0}}(f)}{4 \omega_{0}^{3}}} .
$$

Under the assumption of small damping, $n_{0}^{+} \approx f_{0}$. Then substitute (13) into (11) to derive the FDS expression [8]:

$$
\operatorname{FDS}\left(f_{n}\right)=f_{n} T \frac{K^{b}}{C}\left[\frac{Q \cdot G_{\ddot{Z}}\left(f_{n}\right)}{2\left(2 \pi \cdot f_{n}\right)^{3}}\right]^{b / 2} \Gamma\left(1+\frac{b}{2}\right),
$$




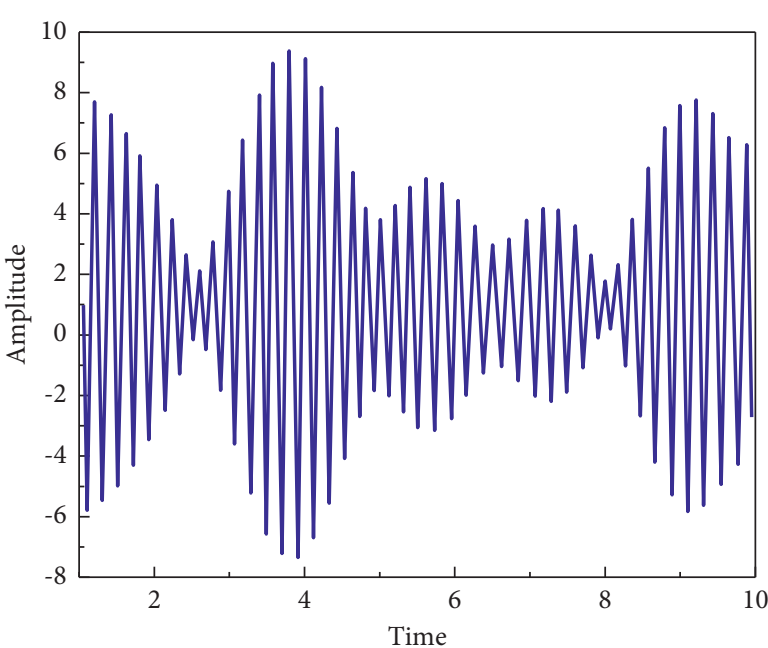

(a)

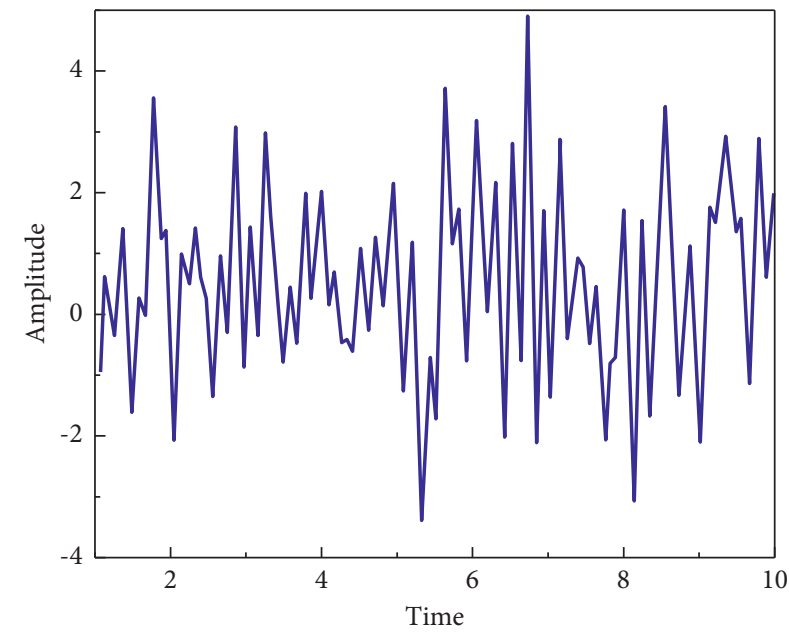

(b)

FiguRe 1: Comparison of wide and narrow band time domain signal waveforms. (a) Narrow band random vibration. (b) Broad band random vibration.

where $f_{n}$ is the system inherent frequency, $K$ is the SDOF system stiffness, $b$ and $C$ are material parameters, and $\Gamma$ is the gamma function, defined as $\Gamma(g)=\int_{0}^{\infty} x^{g-1} \cdot e^{-x} \mathrm{~d} x$.

If the stress response obeys a broad-band distribution, i.e., the irregular coefficient $r$ tends to 0 , the Wirsching and Dirlik probability density functions can be used to calculate the broad-band stress FDS [17].

The Wirsching correction fatigue damage formula is expressed in (15) [19]:

$$
\operatorname{FDS}=\lambda_{R} \operatorname{FDS}\left(f_{n}\right)
$$

where $\operatorname{FDS}\left(f_{n}\right)$ is narrow band damage and $\lambda_{R}$ is the correction coefficient of rain flow counting method which is shown as following:

$$
\lambda_{R}(b, r)=A(b)+[1-A(b)][1-\varepsilon]^{B(b)},
$$

where

$$
\left.\begin{array}{l}
A(b)=0.926-0.033 b \\
B(b)=1.587 b-2.323
\end{array}\right\} .
$$

T. Dirlik proposes the following expression for the probability of rainflow half-ranges [20]:

$$
P_{R}(u)=\frac{D_{1}}{A} e^{u / A}+D_{2} \frac{u}{B^{2}} e^{u^{2} / 2 B^{2}}+D_{3} u e^{u^{2} / 2},
$$

where $\quad D_{1}+D_{2}+D_{3}=1, \quad D_{1}=2\left(C-r^{2}\right) / 1+r^{2}, \quad B$ $=r-C-D_{1}^{2} / 1-r-D_{1}+D_{1}^{2}, D_{2}=1-r-D_{1}+D_{1}^{2} / 1-B$, $A=1.25 r-D_{3}-D_{2} B / D_{1}, C=M_{1} / M_{0} \sqrt{M_{2} / M_{4}}$, and $r$ is the irregular coefficient.

Substituting the probability density of Dirlik (18) into original fatigue damage expression (9), the broad-band FDS calculation formula can be derived [8]:

$$
\begin{aligned}
\mathrm{FDS}= & \frac{K^{b}}{C} n_{p}^{+} T z_{\mathrm{rms}}^{b}\left[D_{1} A^{b} \Gamma(1+b)\right. \\
& \left.+2^{b / 2}\left(D_{2} B^{b}+D_{3}\right) \Gamma\left(1+\frac{b}{2}\right)\right],
\end{aligned}
$$

where $z_{\text {rms }}$ is the RMS value of the displacement response and $T$ is the duration.

2.2.2. Non-Gaussian time domain fatigue damage models. If the excitation cannot be characterized by the PSD, such as an unsteady signal or non-Gaussian signal, the relative displacement response of the signal can be obtained only by transient method. For solving the relative displacement response of a SDOF system, Duhamel integral method is generally used which can be generally expressed as follow [21]:

$$
x(t)=\frac{1}{m \omega_{d}} \int_{0}^{t} p(\tau) e^{-\zeta \omega_{n}(t-\tau)} \sin \left[\omega_{d}(t-\tau)\right] \mathrm{d} \tau,
$$

where $p(\tau)$ is random excitation load, $\omega$ is natural circle frequency and $\zeta$ is damping coefficient. Using the Duhamel integral solution method, although the result is the most accurate, the calculation speed is relatively slow. If there are large numbers of non-Gaussian signals points that need to respond calculation, the Duhamel integral method for solving the analytical solution can not meet the requirements.

Smallwood [22] put forward an improved recursive formula of shock response spectrum in 1981, which includes the fast response formula of relative displacement model. The relative displacement response model and the absolute acceleration response model are shown in Figure 2: 


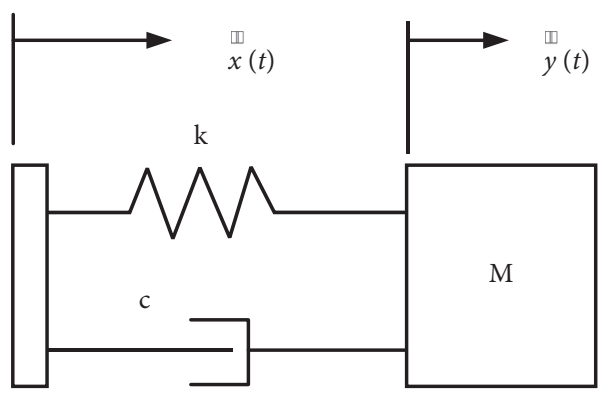

(a)

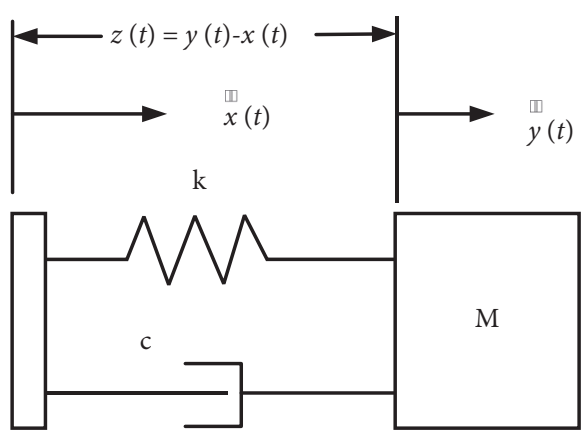

(b)

Figure 2: Response model of foundation vibration. Then the transfer function of the model can be obtained: (a) Absolute acceleration response model. (b) Relative displacement response model.

$$
\widetilde{H}(z)=\frac{b_{0}+b_{1} z^{-1}+b_{2} z^{-2}}{1-2 C z^{-1}+E^{2} z^{-2}} .
$$

The effect of the transfer function can be changed by changing the $b_{0}, b_{1}, b_{2}$, et al. values in the model. So, according to the linear assumption of engineering structures, the stress response can be obtained by equation:

$$
\sigma=K z_{p}
$$

where $\sigma$ is the stress response, $\mathrm{K}$ is the linear multiplier between relative displacement and stress (linear system), and $z_{p}$ is the relative displacement response of the system. The fatigue damage can be obtained by counting the rain flow of the stress response. After calculating the cumulative damage at each natural frequency of the linear system, a frequency damage curve was drawn. Figure 3 shows the detailed flow of solving FDS with non-Gaussian excitation.

\section{Basic principle of frequency-domain synthesis acceleration PSD}

In this study, vibration data of a certain type of airborne equipment, installation bracket, during flight (take-off, climb, flat flight 1 , flat flight 2 , descent, and landing) were used as an excitation input. Aluminum alloy notched specimens were used as research objects to collect the strain data before and after the acceleration bench vibration test. The effectiveness of the accelerated PSD based on different frequency domain synthetic acceleration methods were tested and the whole fatigue life cycle of aluminum alloy notched specimens was detected.

3.1. Measured acceleration excitation input and discrimination. The climbing and flat flying 2 task segments were selected as examples; the distribution test results of them are shown in Figure 4. The kurtosis and skewness of each task segment were calculated, as shown in Figure 5.

The comparative analysis shows that, if the kurtosis is larger than 3.4, the signal can be considered a non-Gaussian signal, while, if the kurtosis is between 3 and 3.4, the signal can be considered a Gaussian signal [23]. In summary, in the mission profile, flat flying 2 corresponds to a Gaussian signal and, because of its large number of sampling points, a fast FDS calculation method can be used later. The other mission segments (take-off, climb, flat flight 1 , descent, and landing) correspond to non-Gaussian signals.

3.2. Selection of acceleration parameters. The FDS of each task segment is calculated first, and the FDS calculation method needs to be chosen according to the load type. Secondly, the appropriate acceleration parameters are selected to calculate the bench test acceleration PSD. The selection of different acceleration parameters has a great influence on the fatigue results of the aluminum alloy notched specimens.

3.2.1. Selection of FDS calculation method. First of all, the effects of different fatigue calculation methods on FDS after the excitation passes through the SDOF system are compared. Taking flat flight 2 signal as an example, the FDS results of the different methods were obtained by programming, as shown in Figure 6.

Based on the RMS values of FDS and accelerated PSD obtained by time domain method, it can be found that the FDS calculated by rainflow method is almost the same and the most accurate, but its calculation speed is slow. The fatigue damage of the simplified Rayleigh distribution has a large float and has more peak points. Because the input excitation is assumed to be white noise for the solution of the RMS value of the relative displacement. The FDSs calculated by the Wirsching and Dirlik methods are very close to those of rainflow counting method. The following analysis compares the differences between these methods from different angles and provides suggestions for selection.

The advantages and disadvantages of various FDS methods are compared from three aspects: load applicability, calculation speed, and calculation accuracy. The speed of calculation is used to evaluate the time spent of synthesis of FDS of each acceleration method. For the evaluation of the calculation accuracy, the relative errors between RMS value of accelerated PSD based on rainflow method and RMS values based on other FDS calculation methods are compared. 


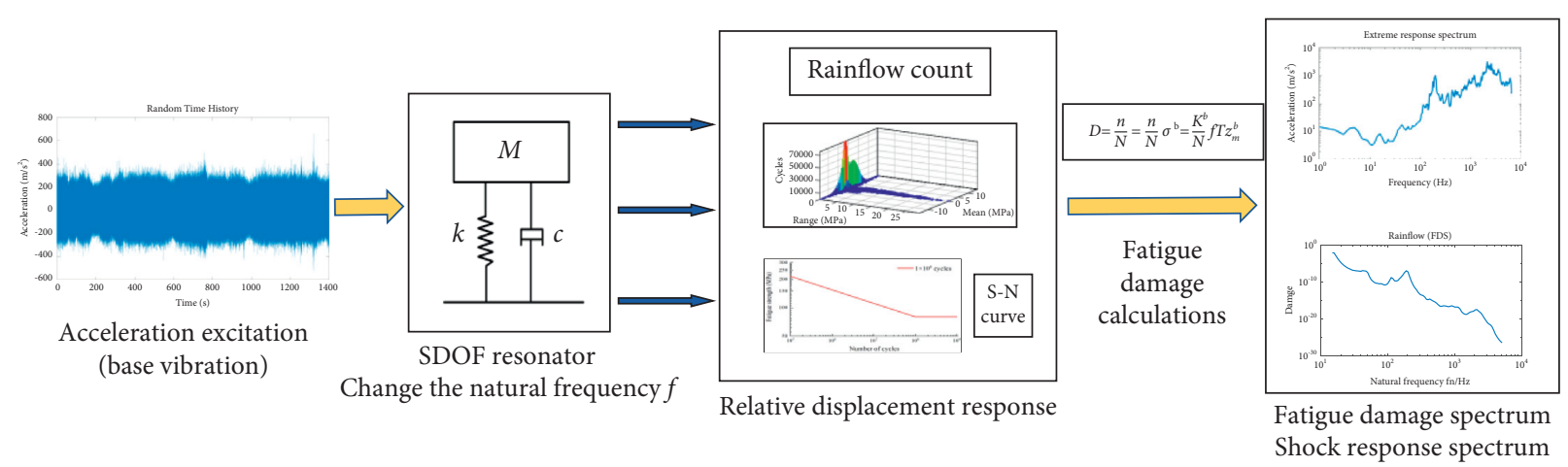

Figure 3: Flow chart of fatigue damage spectrum calculation method under non-Gaussian excitation.

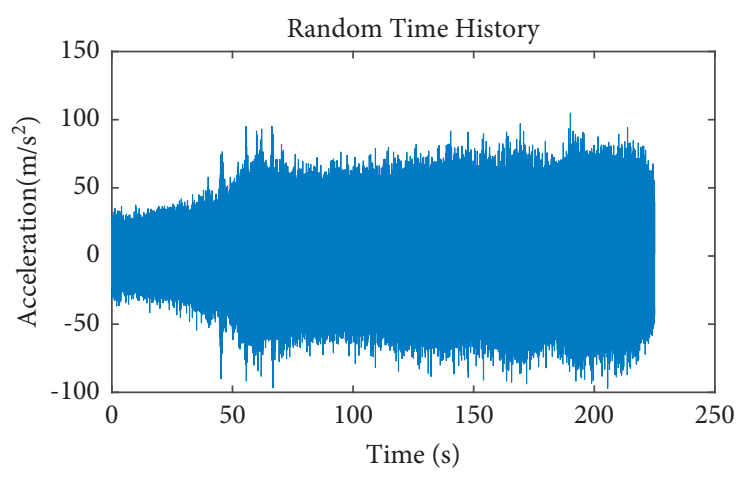

(a)

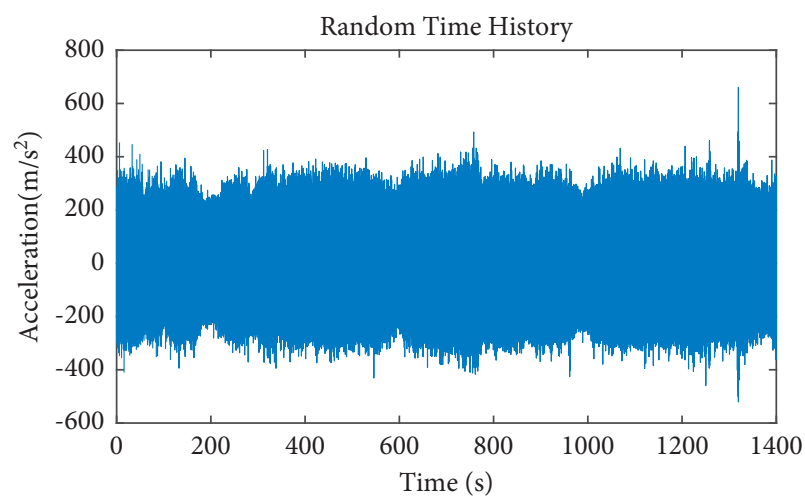

(c)

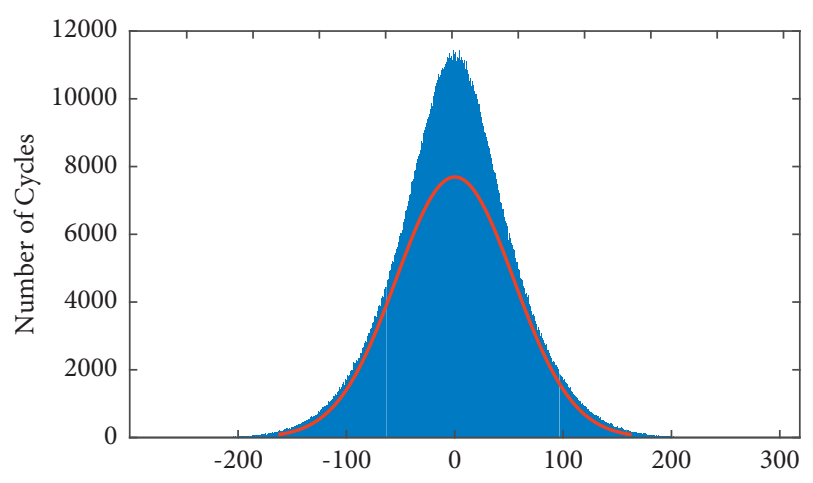

(b)

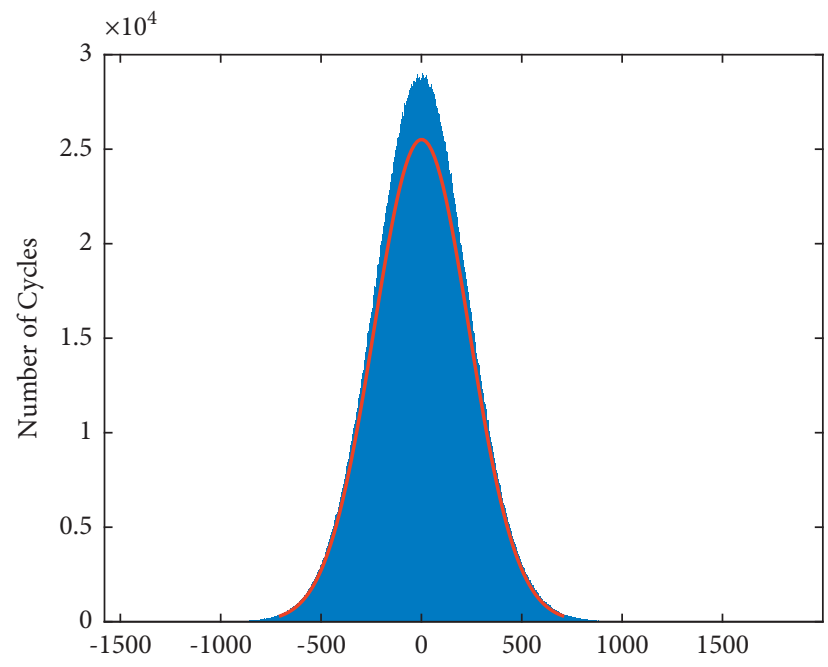

(d)

FIgURe 4: Input Gaussian excitation and non-Gaussian excitation and their amplitude distribution. (a) Climb task segment time-domain acceleration. (b) Time-domain signal distribution inspection of climb task segment. (c) Flat flight 2 task segment time-domain acceleration. (d) Time-domain signal distribution inspection of flight 2.

Table 1 shows that the time-domain rain flow counting result is most accurate, but the calculation is slowest, so it is suitable for non-Gaussian data calculation. The simplified Rayleigh method is fastest and most suitable for the calculation of a large number of points of Gaussian excitation. If the number of sampling points is small, the Dirlik and
Wirsching methods can be used to improve the accuracy of the FDS calculation of Gaussian excitation.

3.2.2. Setting of acceleration parameters. According to the acceleration process, the acceleration parameters can be 


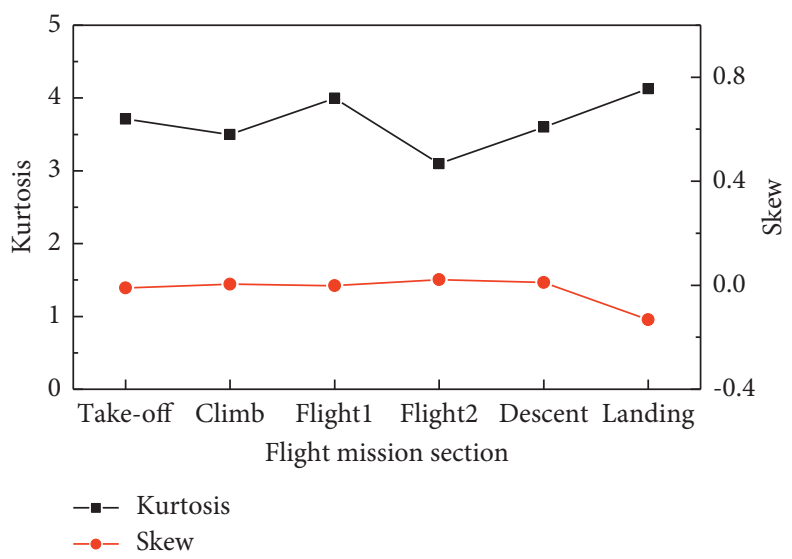

FIgURE 5: Kurtosis and skewness of the input acceleration excitation.

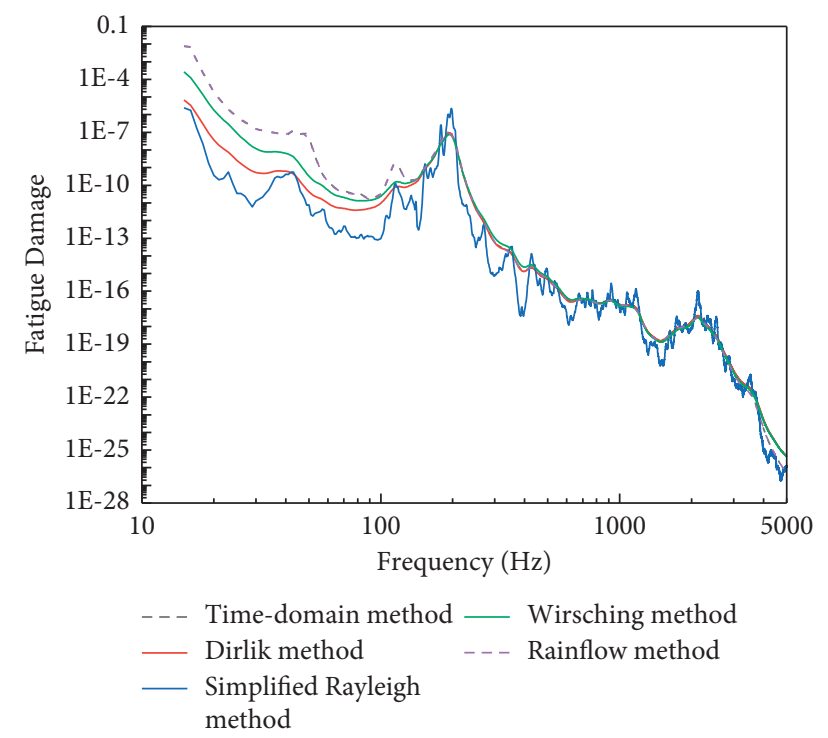

Figure 6: Comparison of FDS calculation results of different methods.

TABLE 1: Multi-dimensional comparison of the advantages and disadvantages of various FDS methods

\begin{tabular}{lccc}
\hline Calculation method & Load applicability & Calculation speed & Calculation accuracy/\% (relative error) \\
\hline Rain flow method & Non-Gaussian, Gaussian & 14 min $27 \mathrm{~s}$ & $/$ \\
Time-domain method & Non-Gaussian, Gaussian & 14 min $9 \mathrm{~s}$ & 0.072 \\
Simplified Rayleigh method & Gaussian & $2 \mathrm{~s}$ & 4.2 \\
Rayleigh method & Gaussian & $8 \mathrm{~s}$ & 5.2 \\
Dirlik method & Gaussian & $10 \mathrm{~s}$ & 3.3 \\
Wirsching method & Gaussian & $10 \mathrm{~s}$ & 0.036 \\
\hline
\end{tabular}

roughly divided into system parameters, material parameters, sampling parameters, FDS estimation parameters, and equivalent acceleration time and so on. The selection of different acceleration parameters has a significant impact on the final acceleration fatigue life. Therefore, in this study, the system parameters (SDOF stiffness $K$ ) and material parameters ( $b, C$ in the $S-N$ curve) are varied to provide the contrastive acceleration PSD, as shown in Figure 7. Meanwhile, by changing the value of $b$, the relationship between preset equivalent acceleration time and pre-simulation accelerate life is shown in Figure 8. It can be seen from these two figures that when the equivalent acceleration time is $1 \mathrm{~h}$ and 


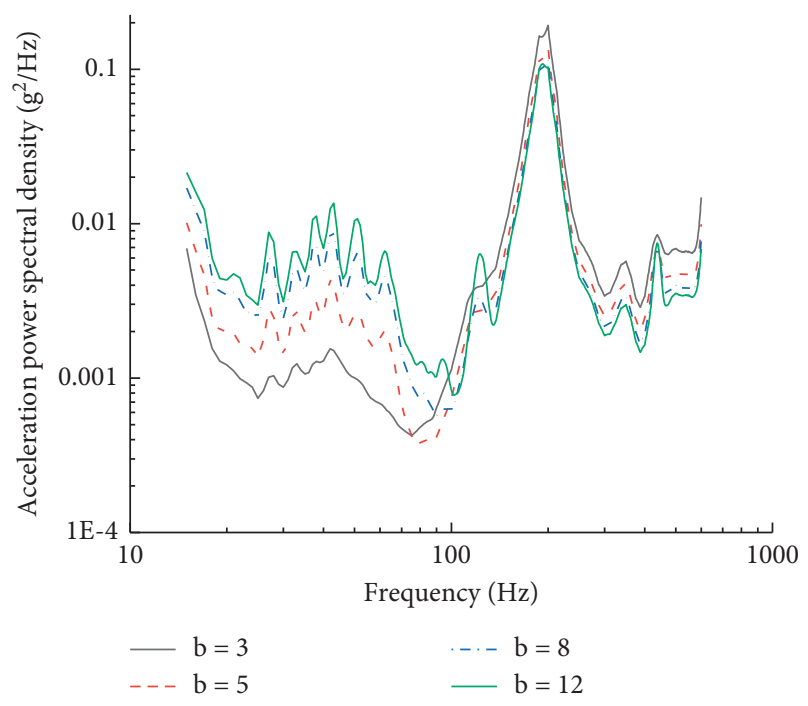

Figure 7: Comparison of PSDs obtained at different acceleration parameters (b).

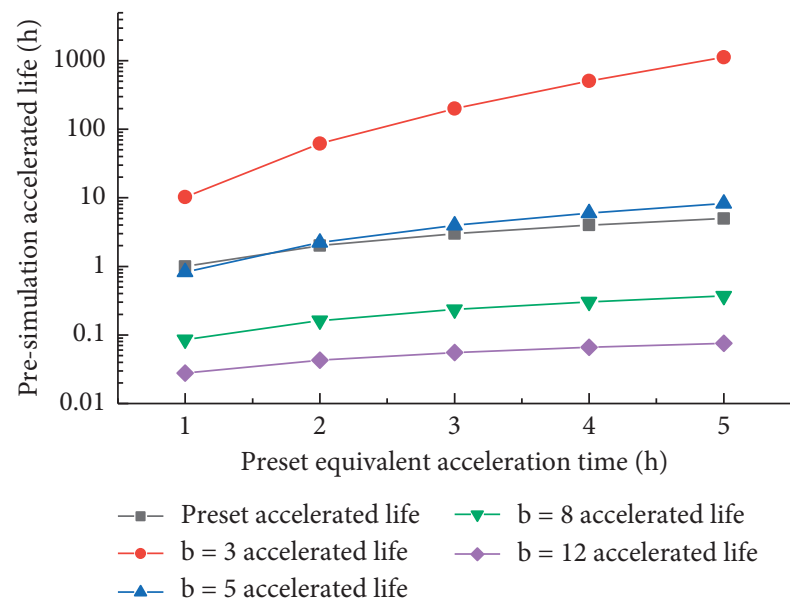

FIGURE 8: Pre-simulation acceleration fatigue lives at different equivalent acceleration time.

the material parameter $b$ is set to 5 , the acceleration fatigue life best-coincides with the preset acceleration fatigue life.

3.3. Establishment of acceleration spectrum. The PSD calculation formula of the synthetic accelerated test within the equivalent time $T_{e q}$ is [24]

$$
G_{\text {synth }}\left(f_{n}\right)=\frac{2\left(2 \pi f_{n}\right)^{3}}{Q}\left[\frac{k \cdot \sum F D S\left(f_{n}\right) \cdot C}{K^{b} f_{n} T_{e q} \Gamma(1+b / 2)}\right]^{2 / b},
$$

where $\sum \operatorname{FDS}\left(f_{n}\right)$ is the total damage of each working condition, $k$ is the safety factor, and $T_{e q}$ is the preset equivalent test time.

Accelerate the non-Gaussian and Gaussian excitation signals of all task segments and take the proportion of both Gaussian and non-Gaussian task phases into account. According to the original fatigue life vibration test results, as shown in section 5.1, each Gaussian and non-Gaussian task phase is assigned with the number of FDS cycles, as shown in Table 2. The accelerated excitation PSD representing the whole life for bench vibration test is shown in Figure 9. A finite-element simulation and bench test verification are carried out as next chapters.

\section{Accelerated fatigue life characteristics of aluminum alloy notched specimens}

4.1. Finite-element model. A one-to-one finite element model was established according to the structure of the specimen and tooling model, as shown in Figure 10. Considering the determination of the patch position, we need to obtain the stress proportion relationship between the patch element and dangerous element, i.e., the stress concentration factor. The simulation provides mainly the fatigue life of the hazard unit, while the strain response of the patch unit was obtained in the bench test. As the acceleration excitation in this study had non-Gaussian characteristics, the quasi-static superposition method and transient response method were used to calculate the original fatigue life, while the uniaxial harmonic response method was used to calculate the accelerated fatigue life. Simultaneously, the large mass point method was used to simulate the input excitation of the shaking table.

The solution method to calculate the stress concentration factor is to add a vertical force of $10 \mathrm{~N}$ at the end of the specimen and evaluate the ratio of the maximum principal stress of the dangerous element to the patch element. The calculated results are shown in Figure 11. The stress concentration factor was 5.3.

4.2. Selection of the $S-N$ curve. In this study, the specimen material was a 7050-T7451 aluminum alloy. According to EN-1999-1-3, the fatigue strength is $140 \mathrm{MPa}$ at a number of $2 \times 10^{6}$ cycles, while the survival rate of the $S-N$ curve was $97.7 \%$, as shown in Figure 12. There are two curves in the figure. The $S$-N curve of $2 \times 10^{6}$ cycles is the fatigue strength of the base metal with defects, and the $S$-N curve of $1 \times 10^{8}$ cycles is the fatigue strength of the base metal without defects. Because the aluminum alloy specimen in this paper has a notch, $2 \times 10^{6}$ is selected as the fatigue cycle.

4.3. Calculation of the Fatigue Damage at the Notch of the Specimen. In this study, all elements at the notch of the aluminum alloy specimen were selected to calculate the fatigue damage. The model transfer function was obtained through the harmonic response analysis based on the modal superposition method and the accelerated PSD excitation was used as the simulation excitation input. The stress response PSD of the model can be obtained using (24). The rain flow amplitude probability density function (PDF), according to the Miner damage accumulation criterion, is obtained using the Dirlik probability density function frequency-domain fatigue model. Finally, the cumulative damage values of all notch risk units are obtained and the fatigue damage calculation formula is presented in (25) [25], 
TABLE 2: Number of FDS cycles that represent the whole life of each mission segment

\begin{tabular}{lccc}
\hline Task phase & Non-Gaussian task segment & Gaussian mission segment $(2.55 g)$ & Gaussian mission segment $(8 g)$ \\
\hline Average number of cycles & 1 & 23.8 & 11.46 \\
\hline
\end{tabular}

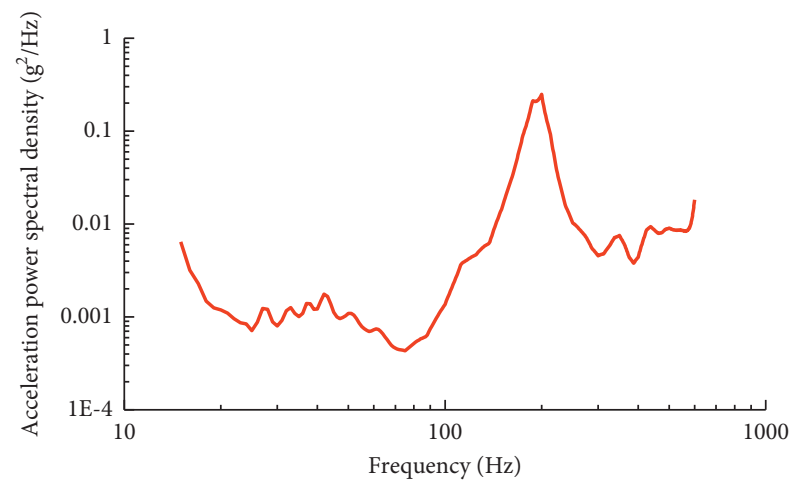

FIGURE 9: PSD of the accelerated test.

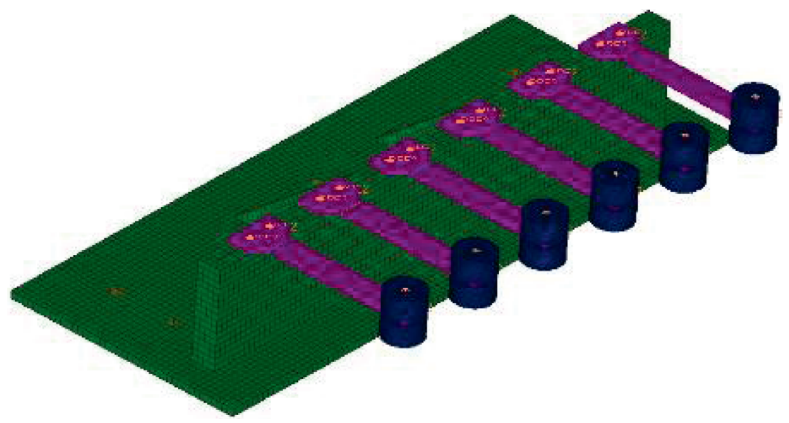

FIGURE 10: Finite-element model of the tooling and aluminum alloy notched specimens.

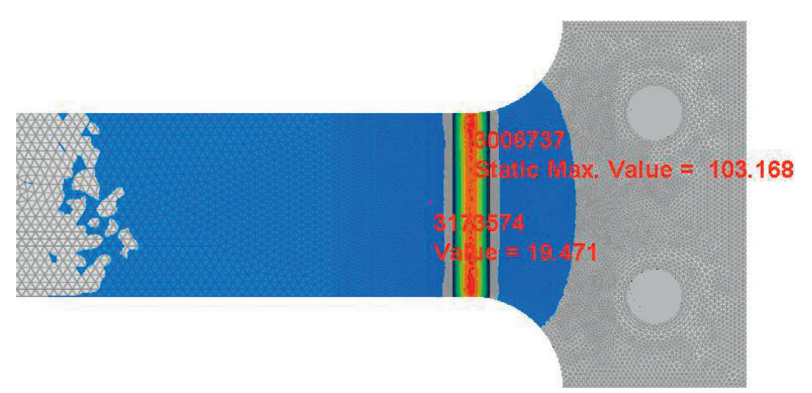

FIgURE 11: Calculation of the stress concentration factor (unit: $\mathrm{MPa})$.

$$
\begin{aligned}
Y\left(f_{n}\right) & =H^{2} \cdot G\left(f_{n}\right), \\
T & =\frac{C}{N_{0} \int_{0}^{+\infty} \sigma^{m} \rho(\sigma) \mathrm{d} \sigma},
\end{aligned}
$$

where $H$ is the transfer function of the model, $G\left(f_{n}\right)$ is the PSD excitation of the input acceleration, $Y\left(f_{n}\right)$ is the PSD response of the system stress, and $\rho(\sigma)$ is the probability density function of the stress amplitude.

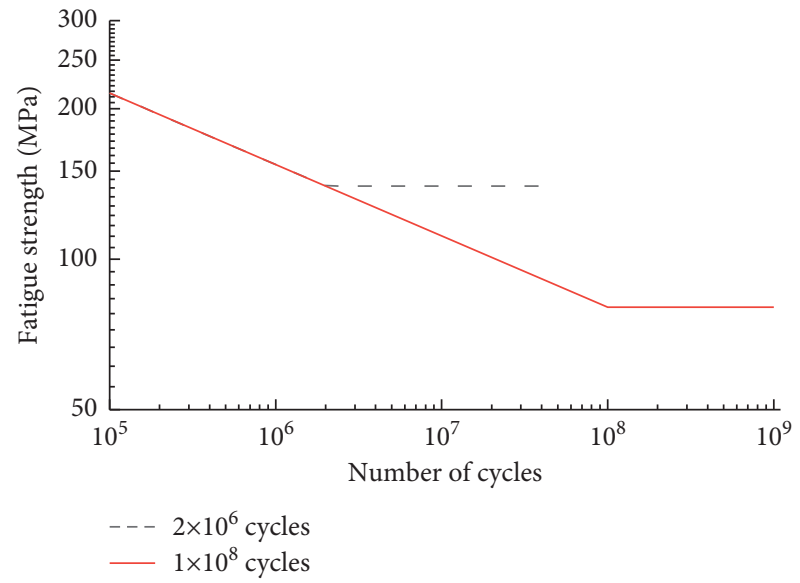

Figure 12: Selection of the $S-N$ curve.

The fatigue damages before and after the acceleration are calculated and compared. The accelerated fatigue damage cloud diagram of the aluminum alloy notched specimen (damage per second) is shown in Figure 13. The selection of the acceleration parameters and fatigue strength during the simulation leads to different acceleration fatigue lives. 


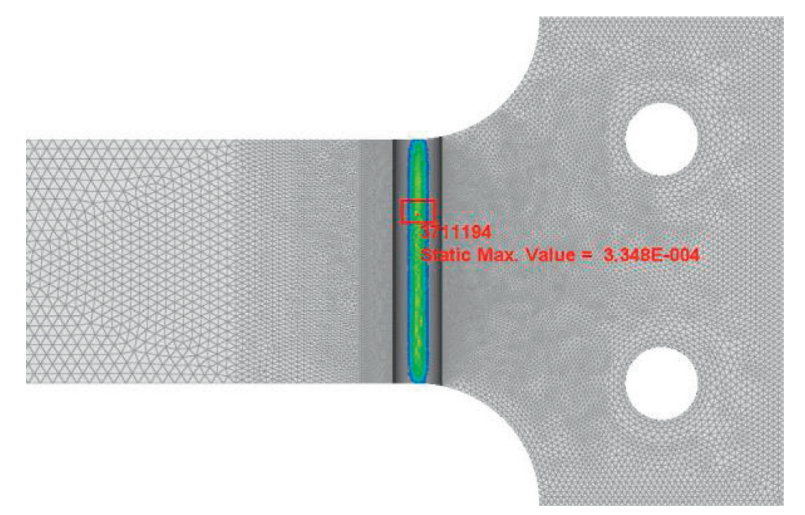

Figure 13: Damage cloud map of the accelerated aluminum alloy notched specimen.

Therefore, to analyze the influences of the acceleration parameters on the accelerated fatigue life of the aluminum alloy notched specimen, Figure 14 compares the damage change law of the notch element with the change in the acceleration parameter $b$. Generally, a larger $b$ led to a larger accelerated damage of the dangerous element. According to a comparative study, the system parameter $K$ and material parameter $C$ affect only the FDS, but do not affect the final accelerated fatigue life [8].

4.4. Comparison of damage before and after the acceleration. Owing to the large number of notch elements, the most dangerous notch element in the simulation analysis is selected to list the fatigue damage calculation results. The simulated cumulative damages before and after the acceleration are compared to the test original fatigue life. The relative error of the calculation is shown in Table 3. The comparative analysis shows that, using the simulation method of uniaxial harmonic response, the calculated original fatigue life is close to the test original fatigue life; the relative error is $15.7 \%$. The relative error between the simulated accelerated cumulative damage and test cumulative damage (calculated at $D=1$ ) is $20.5 \%$. With the uniaxial harmonic response method to calculate the $\mathrm{cu}$ mulative damage, the accelerated damage of the dangerous element is larger than the original damage. The error is related to the calculation method of the original fatigue life and linear accumulation of damage and is within the allowable range, which verifies the effectiveness of the accelerated method used in this study.

\section{Bench test verification}

5.1. Original fatigue life vibration test. As shown in the previous sections, the input contains Gaussian and nonGaussian excitations. To obtain the original fatigue life of the specimen under the mixed excitation, i.e. to obtain the FDS cycle times representing the whole life, the original fatigue life test was carried out. The test table layout and specimen number are shown in Figures 15 and 16

Firstly, non-Gaussian excitations were applied to the notched aluminum alloy specimen in the form of a

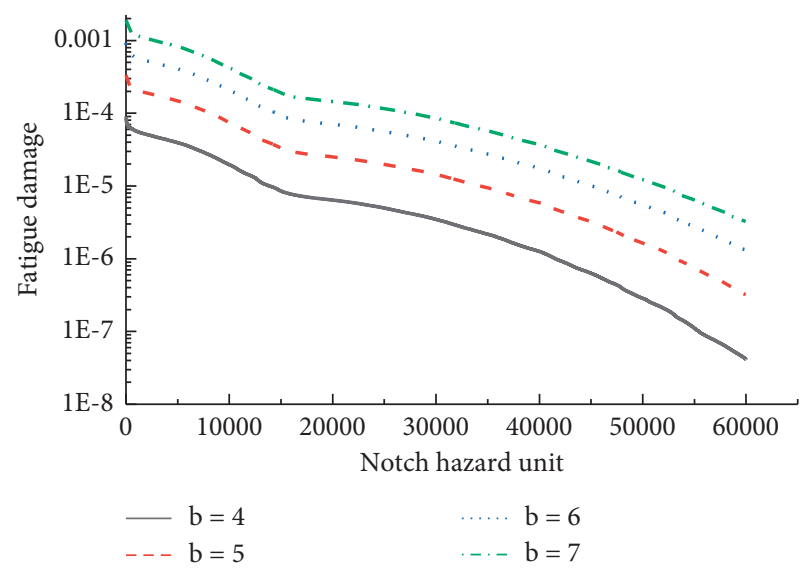

FIgURE 14: Damage comparison by the harmonic response method (change in acceleration parameter (b)).

time-domain waveform representation, and then Gaussian PSD excitations with different effective values are applied until the fracture of the specimen, and the fracture time is recorded. The duration of non-Gaussian vibration is $44 \mathrm{~min}$, while the RMS valid values of the Gaussian PSD excitation were $2.55 \mathrm{~g}$ and $8 \mathrm{~g}$. The original fatigue life test results are listed in Table 4.

The average original fatigue life of the aluminum alloy notched specimen was $42095 \mathrm{~s}$. The purpose of the original fatigue life vibration test is to obtain the fatigue life of aluminum alloy notched specimens under Gaussian and non-Gaussian mixed excitation, so as to calculate the number of cycles of FDS which represents the whole life.

5.2. Vibration endurance acceleration test. To further verify the effectiveness of the random vibration excitation acceleration method used in this study, uniaxial original spectrum time-domain simulation tests and vibration endurance acceleration tests were carried out. A group of six aluminum alloy notch specimens were tested. Strain gauges were pasted near the notches on the upper surface of the specimens, strain responses were measured, and accelerometers were installed on the shaking table to compare the consistency of input and output excitations. In this experiment, six unidirectional strain gauges and two accelerometers were used in a total of 12 channels. Using the crack time as a reference, the failure time of the specimen was recorded and the strain was measured. The test table is arranged as shown in Figure 17. The fracture fatigue life of specimens was recorded and the measured strain cumulative life was calculated according to the strain signal, as shown in Table 5. The relative errors of test (calculated by preset equivalent acceleration time and crack initiation fatigue life) and the relative errors of simulation (calculated by measured strain cumulative life and simulation cumulative life) were calculated. At the same time, the mean values of the absolute relative errors were computed. Finally, calculate the frequency domain characteristics of the stress signal at the dangerous point of each specimen and compare the relative error between the mean value of test life and the mean value of simulation life, as shown in Table 6 . 
TABle 3: Comparison of cumulative damages before and after the acceleration $(b=5)$

\begin{tabular}{lccc}
\hline Hazard unit & & 3711194 & Test \\
\hline Cumulative damage & Original cumulative damage & Accelerated cumulative damage & Original damage \\
Relative error/\% & 0.8431 & 1.205 & 1 \\
Fatigue life $(\mathrm{h})$ & 15.7 & 20.5 & $/$ \\
& Original cumulative life & Accelerated cumulative life & Original fatigue life \\
\end{tabular}

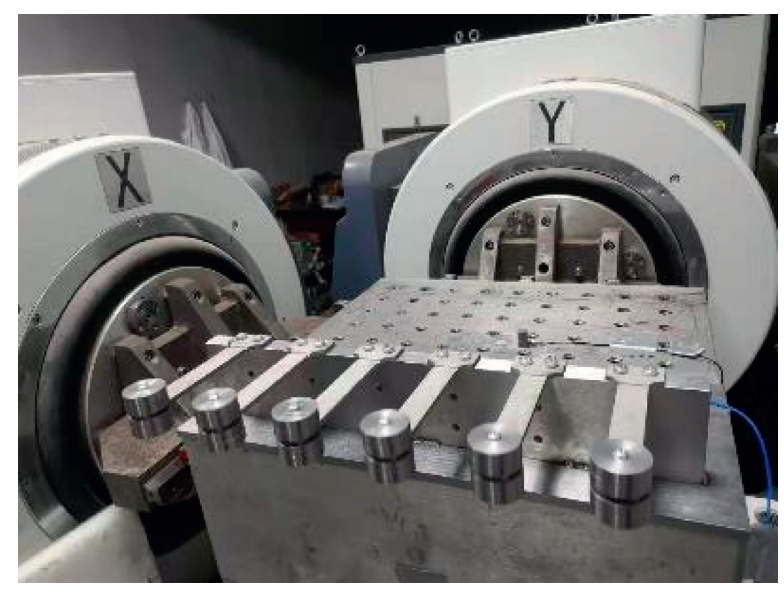

Figure 15: Original fatigue life test countertop arrangement.

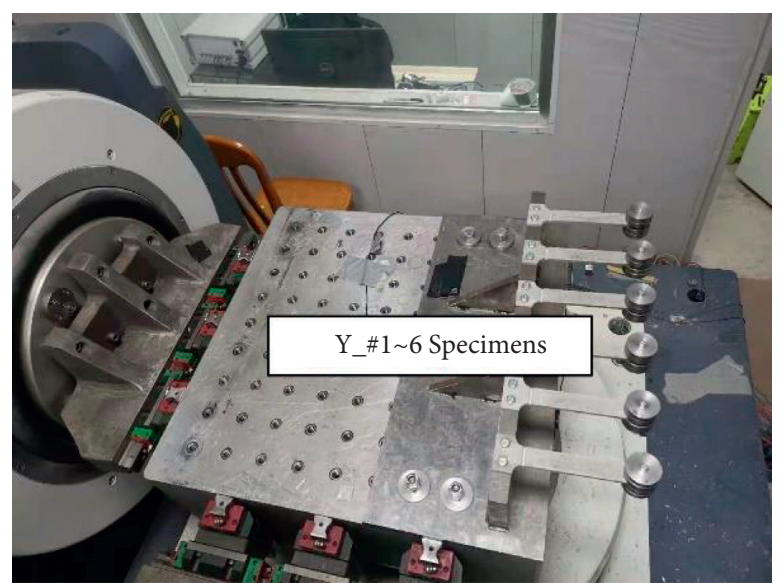

Figure 16: Original fatigue life test sample number.

TABLE 4: Original fatigue life test results

\begin{tabular}{|c|c|c|c|c|c|c|c|c|}
\hline \multirow{3}{*}{$\begin{array}{l}\text { Test specimen } \\
\text { number }\end{array}$} & \multicolumn{7}{|c|}{ Mission profile } & \multirow{3}{*}{$\begin{array}{l}\text { Total time } \\
\text { (s) }\end{array}$} \\
\hline & \multicolumn{5}{|c|}{ Non-Gaussian task segment (s) } & \multicolumn{2}{|c|}{ Gaussian task section (s) } & \\
\hline & $\begin{array}{c}\text { Take- } \\
\text { off }\end{array}$ & Climb & Flat flight & Descent & Landing & $\begin{array}{c}\text { Gaussian excitation } \\
(2.55 \mathrm{~g})\end{array}$ & $\begin{array}{c}\text { Gaussian excitation } \\
(8 g)\end{array}$ & \\
\hline Y1_1 & 32 & 240 & 1493 & 864 & 16 & 34200 & 4140 & 40985 \\
\hline Y1_2 & 32 & 240 & 1493 & 864 & 16 & 34200 & 6600 & 43445 \\
\hline Y1_3 & 32 & 240 & 1493 & 864 & 16 & 34200 & 4860 & 41705 \\
\hline Y1_4 & 32 & 240 & 1493 & 864 & 16 & 34200 & 4080 & 40925 \\
\hline Y1_5 & 32 & 240 & 1493 & 864 & 16 & 34200 & 4740 & 41585 \\
\hline Y1_6 & 32 & 240 & 1493 & 864 & 16 & 34200 & 7080 & 43925 \\
\hline
\end{tabular}




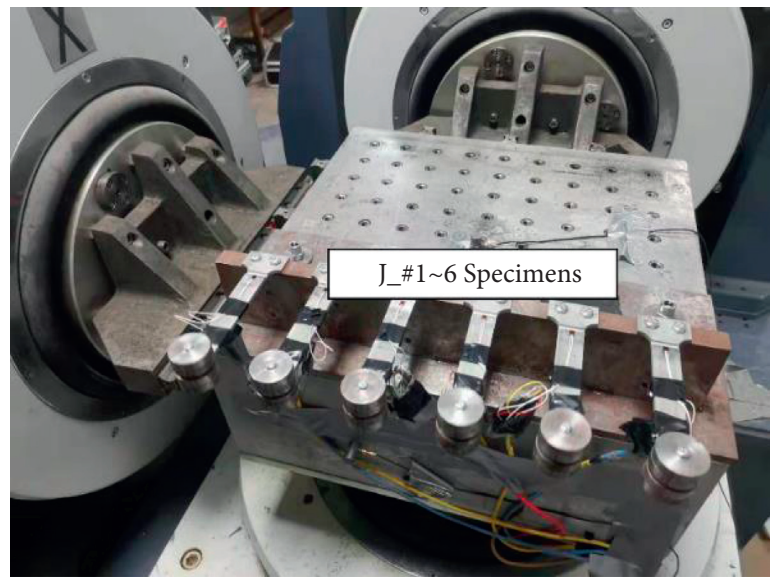

FIGURE 17: Sample number of the vibration endurance accelerated test.

TABLE 5: Comparison of the accelerated fatigue life between the bench test and simulation

\begin{tabular}{|c|c|c|c|c|c|c|}
\hline $\begin{array}{l}\text { Test } \\
\text { piece }\end{array}$ & $\begin{array}{c}\text { Preset equivalent } \\
\text { acceleration time }(\mathrm{min})\end{array}$ & $\begin{array}{l}\text { Crack initiation } \\
\text { fatigue life (min) }\end{array}$ & $\begin{array}{c}\text { Measured strain } \\
\text { cumulative life (min) }\end{array}$ & $\begin{array}{c}\text { Simulation } \\
\text { cumulative life } \\
(\min )\end{array}$ & $\begin{array}{c}\text { Relative } \\
\text { error/\% (test) }\end{array}$ & $\begin{array}{c}\text { Relative error/ } \\
\% \\
\text { (simulation) }\end{array}$ \\
\hline 1 & 60 & 67 & 42.708 & 51.8 & 11.67 & 21.29 \\
\hline 2 & 60 & 52 & 34.43 & 51.9 & -13.33 & 50.74 \\
\hline 3 & 60 & 73 & 49.524 & 49.8 & 21.67 & 0.56 \\
\hline 4 & 60 & 63 & 34.776 & 49.8 & 5.00 & 43.20 \\
\hline 5 & 60 & 68 & 47.436 & 51.7 & 13.33 & 8.99 \\
\hline 6 & 60 & 82 & 86.9 & 51.7 & 36.67 & -40.51 \\
\hline mean & 60 & 67.5 & 49.296 & 51.117 & 16.95 & 27.55 \\
\hline
\end{tabular}

TABle 6: Calculation of frequency domain characteristics of stress signal and comparison of fatigue life between test and simulation

\begin{tabular}{|c|c|c|c|c|c|c|}
\hline \multirow{2}{*}{ Hazard unit } & \multicolumn{6}{|c|}{3711194} \\
\hline & S1 & S2 & S3 & S4 & S5 & S6 \\
\hline 0th spectral moment & 196.09 & 125.96 & 123.77 & 172.51 & 204.07 & 39.07 \\
\hline 1 th spectral moment & $6.58 \mathrm{e} 3$ & $4.33 \mathrm{e} 3$ & $4.35 \mathrm{e} 3$ & $5.78 \mathrm{e} 3$ & $6.88 \mathrm{e} 3$ & $1.41 \mathrm{e} 3$ \\
\hline 2nd spectral moment & $2.65 \mathrm{e} 5$ & $2.12 \mathrm{e} 5$ & $2.82 \mathrm{e} 5$ & $2.9 \mathrm{e} 5$ & $2.96 \mathrm{e} 5$ & $1.03 \mathrm{e} 5$ \\
\hline 4 th spectral moment & $9.33 \mathrm{e} 9$ & $1.35 \mathrm{e} 10$ & $1.35 \mathrm{e} 11$ & $1.81 \mathrm{e} 11$ & $1.37 \mathrm{e} 10$ & $7.79 \mathrm{e} 10$ \\
\hline Peaks per second & 187.47 & 252.82 & 692.49 & 790.19 & 215.6 & 868.05 \\
\hline Upward mean crossings per second & 36.8 & 41.06 & 47.69 & 41.03 & 38.1 & 51.44 \\
\hline Irregularity factor & 0.1963 & 0.1624 & 0.0689 & 0.0519 & 0.1767 & 0.0593 \\
\hline Fatigue life (min) & \multicolumn{2}{|c|}{$\begin{array}{l}\text { Simulation } \\
51.117\end{array}$} & \multicolumn{2}{|c|}{$\begin{array}{l}\text { Test } \\
67.5\end{array}$} & \multicolumn{2}{|c|}{$\begin{array}{c}\text { Relative error/\% } \\
24.27\end{array}$} \\
\hline
\end{tabular}

Through the bench vibration tests and finite-element simulation, and the comparison of all kinds of fatigue life, the accelerated fatigue life in the bench test is slightly larger than the preset equivalent accelerated fatigue life. The average accelerated fatigue life is $67.5 \mathrm{~min}$, while the relative error with respect to the preset equivalent acceleration fatigue life is $16.95 \%$. The average relative error between the measured strain accumulation and accelerated fatigue life of the finite-element simulation is $27.55 \%$. The causes of the error are the difference of finite element model and the difference of fatigue calculation methods. In the bench test and finite-element simulation, the average relative error is within the allowable limit. The effectiveness of the nonGaussian acceleration method and accuracy of the finiteelement accelerated fatigue life simulation method were verified.
5.3. Acceleration ideal factor calculation. The acceleration ideal factor is used to define the acceleration effect. The ideal acceleration factor is equal to the failure fatigue life of specimens divided by the equivalent acceleration time. A closer acceleration ideal factor to 1 implies a better acceleration effect. An acceleration ideal factor $>>1$ indicates that the acceleration excitation does not reflect the long-life operation of the equipment within the effective time. If the acceleration ideal factor is close to 0 , the acceleration is excessive. The calculation of the ideal acceleration factor is shown in Figure 18. The ideal factor of the test acceleration is slightly larger than the simulation result owing to the cumulative results of strain damage, which is related to the temperature change in the test and control accuracy of the shaking table. The randomness of the test is relatively large. The average acceleration ideal factor is 1.125 , indicating that 


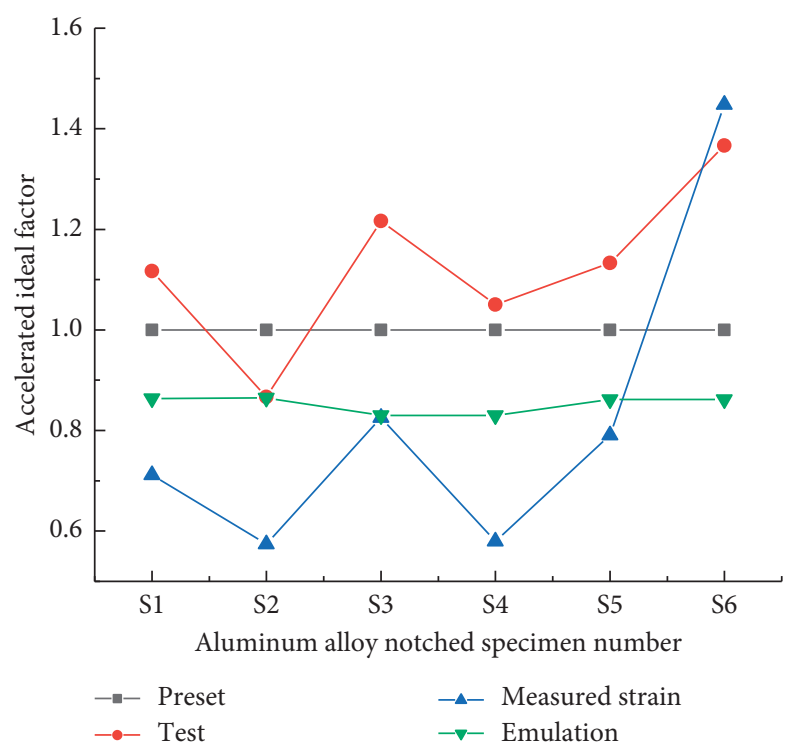

FIgURE 18: Comparison of ideal factors of the bench test and simulation acceleration.

the acceleration is not excessive. The excitation can reflect the total service life of the specimen at a specified time.

\section{Conclusion}

In this study, an acceleration method to address nonGaussian excitation is proposed based on the fatigue damage spectrum theory. The aluminum alloy notched specimen is designed and the bench test and finite element simulation are carried out at the same time to verify the effectiveness of the method.

(1) The calculation formulas of fatigue damage spectrum under Gaussian excitation and non-Gaussian excitation are derived, and the calculation flow chart of fatigue damage spectrum by acceleration excitation rain flow counting method is drawn. Simultaneously A discrimination method for non-Gaussian excitation was proposed and the kurtosis and skewness of each task segment were calculated. The Gaussian distribution characteristics of each task segment were tested and an amplitude distribution map was drawn. The Flat Flying 2 mission segment corresponded to a Gaussian excitation, while the rest of the task segment to a non-Gaussian excitation.

(2) The effects of different FDS methods on the acceleration spectra were analyzed. The simplified Rayleigh method was most suitable for the calculation of a large number of data points. The Dirlik calculation accuracy was highest. The rain flow counting method was suitable for non-Gaussian loads. The acceleration effect was best when the acceleration parameter was $b=5$ and the equivalent acceleration time was $1 \mathrm{~h}$.

(3) The finite-element model of the aluminum alloy notched specimen and tooling was established. The stress concentration factor was 5.3. The fatigue lives before and after the acceleration were calculated by the uniaxial harmonic response method and compared to the original test life. The simulation results showed that the relative error of the original $\mathrm{cu}$ mulative damage of the dangerous element was $15.7 \%$. The relative error of the accelerated cumulative damage was $20.5 \%$. The changes in the acceleration parameters revealed that a higher $b$ led to a larger accelerated cumulative damage.

(4) The original fatigue life vibration test and accelerated fatigue life test were carried out respectively. The bench test results of the original fatigue life show that the number of non-Gaussian FDS cycles is 1 , and the number of Gaussian FDS cycles is 23.8 and 11.46 respectively. The bench test results of accelerated fatigue life show that the relative error of fatigue life before and after acceleration is less than $16.95 \%$, and the relative error of test and simulation is $24.27 \%$. The failure time of the specimen accelerated from approximately $12 \mathrm{~h}$ to $1 \mathrm{~h}$; the acceleration ratio reached 12 . The acceleration method can process the signal with non-Gaussian and Gaussian excitations into an accelerated PSD as a bench test-simulation input. The purpose of this study was to provide a reference for the compilation of the load spectrum and vibration endurance acceleration test of other equipment in aviation.

\section{Data Availability}

The data used to support the findings of this study are available from the corresponding author upon request.

\section{Conflicts of Interest}

The authors declare no conflicts of interest.

\section{Acknowledgments}

This study was financially supported by the China Aeronautical Science Foundation (20180241001).

\section{References}

[1] H.-j. Kim, B.-S. Jang, and J. D. Kim, "Fatigue-damage prediction for ship and offshore structures under wide-banded non-Gaussian random loadings part I: approximation of cycle distribution in wide-banded Gaussian random processes," Applied Ocean Research, vol. 101, Article ID 102294, 2020.

[2] Yu Jiang, J. Tao, and X. Chen, "Super-Gaussian random vibration fatigue accelerated testing model," Journal of Vibration and Shock, vol. 36, no. 9, pp. 261-266, 2017.

[3] A. Angeli, B. Cornelis, and M. Troncossi, "Synthesis of sineon-random vibration profiles for accelerated life tests based on fatigue damage spectrum equivalence," Mechanical Systems and Signal Processing, vol. 103, pp. 340-351, 2018.

[4] Y. Liu, Research on Accelerated Fatigue Life Testing Technology of Mechanical Structure under Super-gaussian Vibration Excitation, National University of Defense Technology, Zuny, China, 2015. 
[5] H. Chen, Research on the Fatigue Life and Reliability of Mechanical Structure under Non-gaussian Random Loading, National University of Defense Technology, Zuny, China, 2014.

[6] J. Wang, Fatigue Prediction and Accelerated Fatigue Test of Multiaxial Random Vibration, Southwest Jiaotong University, Chengdu, China, 2019.

[7] W. Qin, P. Wang, Y. Liu, and T. Xindi, "Random vibration acceleration test method of CI cabinet based on FDS [J/OL]," Equipment Environmental Engineering, vol. 1-8, 2021, http:// kns.cnki.net/kcms/detail/50.1170.X.20200828.1551.002.html.

[8] C. Lalanne, "Mechanical vibration and shock analysis," Specification Development, John Wiley \& Sons, Hoboken, NY, USA, 2013.

[9] P. Wolfsteiner and A. Trapp, "Fatigue life due to nonGaussian excitation - an analysis of the fatigue damage spectrum using higher order spectra," International Journal of Fatigue, vol. 127, pp. 203-216, 2019.

[10] C. Wen, B. Xie, Z. Li, Y. Yin, X. Zhao, and Z. Song, "Power density based fatigue load spectrum editing for accelerated durability testing for tractor front axles," Biosystems Engineering, vol. 200, pp. 73-88, 2020.

[11] F. Cianetti, A. Alvino, A. Bolognini, M. Palmieri, and C. Braccesi, "On field durability tests of mechanical systems. the use of the fatigue damage spectrum," Procedia Structural Integrity, vol. 3, pp. 176-190, 2017.

[12] Q. Li, G. Chen, M. Wang, and H. Yao, "Study on vibration acceleration factor test method," Journal of Vibration, Measurement \& Diagnosis, vol. 33, no. 1, pp. 35-39+163, 2013.

[13] W. L. Fan, "Statistics analysis for response of linear structure under stationary non-Gaussian excitation based on the higher-order pseudo-excitation method," China Civil Engineering Journal, vol. 52, no. 10, pp. 30-35, 2019.

[14] S. Gao, Non-Gaussian Response Extrema and Fatigue of Marine Structures, Dalian University of Technology, Dalian, China, 2019.

[15] A. Steinwolf, "Random vibration testing beyond PSD limitations," Sound and Vibration, vol. 40, no. 9, pp. 12-21, 2006.

[16] E. Pesaresi and M. Troncossi, "Synthesis of vibration signals with prescribed power spectral density and kurtosis value," in Proceedings of the ISMA2018 International Conference on Noise and Vibration Engineering, ISMA, Leuven, Belgium, September 2018.

[17] Y. E. Fei, Research on Fatigue Life for Structures under Random Vibration Loading, Tianjin University, TianJin, China, 2017.

[18] A. Papoulis, "Narrow-band systems and gaussianity," IEEE Transactions on Information Theory, vol. 18, no. 1, pp. 20-27, 1972.

[19] S. R. Win t' erstein, "Nonlinear vibration models for extremes and fatigue," Journal of Engineering Mechanics, vol. 114, no. 10, pp. 1772-1790, 1988.

[20] T. Dirlik, Application of Computers in Fatigue Analysis, School of Engineering, University of Warwick, Coventry, UK, 1985.

[21] F. Kihm, A. Halfpenny, and K. Munson, "Synthesis of accelerated and more realistic vibration endurance tests using kurtosis," in Proceedings of the SAE Technical Paper, Detroit, MI, USA, April 2016.

[22] D. O. Smallwood, "An improved recursive formula for calculating shock response spectra," Shock and Vibration Bulletin, vol. 51, no. 2, pp. 211-217, 1981.

[23] J. H. E. Wu, F. W. Zhiguo, Non-Gaussian random vibration and kurtosis control," Structure \& Environment Engineering, vol. 45, no. 6, pp. 1-9, 2018.
[24] A. Halfpenny, Accelerated Vibration Testing Based On Fatigue Damage Spectra, nCode International, Sheffield, UK, 2006.

[25] X. Lin, "Fatigue life estimation based on power spectral density signals," China Mechanical Engineering, vol. 2, no. 11, pp. 20-23+2, 1998. 